\title{
Two Remarks to Bifullness of Centers of Archimedean Atomic Lattice Effect Algebras
}

\author{
M. Kalina
}

\begin{abstract}
Lattice effect algebras generalize orthomodular lattices as well as MV-algebras. This means that within lattice effect algebras it is possible to model such effects as unsharpness (fuzziness) and/or non-compatibility. The main problem is the existence of a state. There are lattice effect algebras with no state. For this reason we need some conditions that simplify checking the existence of a state. If we know that the center $C(E)$ of an atomic Archimedean lattice effect algebra $E$ (which is again atomic) is a bifull sublattice of $E$, then we are able to represent $E$ as a subdirect product of lattice effect algebras $E_{i}$ where the top element of each one of $E_{i}$ is an atom of $C(E)$. In this case it is enough if we find a state at least in one of $E_{i}$ and we are able to extend this state to the whole lattice effect algebra $E$. In [8] an atomic lattice effect algebra $E$ (in fact, an atomic orthomodular lattice) with atomic center $C(E)$ was constructed, where $C(E)$ is not a bifull sublattice of $E$. In this paper we show that for atomic lattice effect algebras $E$ (atomic orthomodular lattices) neither completeness (and atomicity) of $C(E)$ nor $\sigma$-completeness of $E$ are sufficient conditions for $C(E)$ to be a bifull sublattice of $E$.
\end{abstract}

Keywords: lattice effect algebra, orthomodular lattice, center, atom, bifullness.

\section{Preliminaries}

Effect algebras, introduced by D. J. Foulis and M. K. Bennett [3], have their importance in the investigation of uncertainty. Lattice ordered effect algebras generalize orthomodular lattices and MValgebras. Thus they may include non-compatible pairs of elements as well as unsharp elements.

Definition 1 (Foulis and Bennett [3]) An effect algebra is a system $(E ; \oplus, \mathbf{0}, \mathbf{1})$ consisting of a set $E$ with two different elements $\mathbf{0}$ and $\mathbf{1}$, called zero and unit, respectively and $\oplus$ is a partially defined binary operation satisfying the following conditions for all $p, q, r \in E$ :

(E1) If $p \oplus q$ is defined, then $q \oplus p$ is defined and $p \oplus q=q \oplus p$.

(E2) If $q \oplus r$ is defined and $p \oplus(q \oplus r)$ is defined, then $p \oplus q$ and $(p \oplus q) \oplus r$ are defined and $p \oplus(q \oplus r)=(p \oplus q) \oplus r$.

(E3) For every $p \in E$ there exists a unique $q \in E$ such that $p \oplus q$ is defined and $p \oplus q=\mathbf{1}$.

(E4) If $p \oplus \mathbf{1}$ is defined then $p=\mathbf{0 .}$

The element $q$ in (E3) will be called the supplement of $p$, and will be denoted as $p^{\prime}$.

In the whole paper, for an effect algebra $(E, \oplus, \mathbf{0}, \mathbf{1})$, writing $a \oplus b$ for arbitrary $a, b \in E$ will mean that $a \oplus b$ exists. On an effect algebra $E$ we may define another partial binary operation $\ominus$ by

$$
a \ominus b=c \quad \Leftrightarrow \quad b \oplus c=a .
$$

The operation $\ominus$ induces a partial order on $E$.
Namely, for $a, b \in E b \leq a$ if there exists a $c \in E$ such that $a \ominus b=c$. If $E$ with respect to $\leq$ is lattice ordered, we say that $E$ is a lattice effect algebra. For the sake of brevity we will write just LEA. Further, in this article we often briefly write 'an effect algebra $E$ ' skipping the operations.

S. P. Gudder $([5,6])$ introduced the notion of sharp elements and sharply dominating lattice effect algebras. Recall that an element $x$ of the LEA $E$ is called sharp if $x \wedge x^{\prime}=\mathbf{0}$. Jenča and Riečanová in [7] proved that in every lattice effect algebra $E$ the set $S(E)=\left\{x \in E ; x \wedge x^{\prime}=\mathbf{0}\right\}$ of sharp elements is an orthomodular lattice which is a sub-effect algebra of $E$, meaning that if among $x, y, z \in E$ with $x \oplus y=z$ at least two elements are in $S(E)$ then $x, y, z \in S(E)$. Moreover $S(E)$ is a full sublattice of $E$, hence a supremum of any set of sharp elements, which exists in $E$, is again a sharp element. Further, each maximal subset $M$ of pairwise compatible elements of $E$, called a block of $E$, is a sub-effect algebra and a full sublattice of $E$ and $E=\bigcup\{M \subseteq E ; M$ is a block of $E\}$ (see $[16,17])$. Central elements and centers of effect algebras were defined in [4]. In $[14,15]$ it was proved that in every lattice effect algebra $E$ the center

$$
\begin{aligned}
C(E) & =\left\{x \in E ;(\forall y \in E) y=(y \wedge x) \vee\left(y \wedge x^{\prime}\right)\right\} \\
& =S(E) \cap B(E),
\end{aligned}
$$

where $B(E)=\bigcap\{M \subseteq E ; M$ is a block of $E\}$. Since $S(E)$ is an orthomodular lattice and $B(E)$ is an 
MV-effect algebra, we obtain that $C(E)$ is a Boolean algebra. Note that $E$ is an orthomodular lattice if and only if $E=S(E)$ and $E$ is an MV-effect algebra if and only if $E=B(E)$. Thus $E$ is a Boolean algebra if and only if $E=S(E)=B(E)=C(E)$.

Recall that an element $p$ of an effect algebra $E$ is called an atom if and only if $p$ is a minimal nonzero element of $E$ and $E$ is atomic if for each $x \in E$, $x \neq \mathbf{0}$, there exists an atom $p \leq x$.

Definition 2 Let $(E, \oplus, 0)$ be an effect algebra. To each $a \in E$ we define its isotropic index, notation ord $(a)$, as the maximal positive integer $n$ such that

$$
n a:=\underbrace{a \oplus \ldots \oplus a}_{n \text {-times }}
$$

exists. We set $\operatorname{ord}(a)=\infty$ if na exists for each positive integer $n$. We say that $E$ is Archimedean, if for each $a \in E, a \neq \mathbf{0}, \operatorname{ord}(a)$ is finite.

An element $u \in E$ is called finite, if there exists a finite system of atoms $a_{1}, \ldots, a_{n}$ (which are not necessarily distinct) such that $u=a_{1} \oplus \ldots \oplus a_{n}$. An element $v \in E$ is called cofinite, if there exists a finite element $u \in E$ such that $v=u^{\prime}$.

We say that for a finite system $F=\left(x_{j}\right)_{j=1}^{k}$ of not necessarily different elements of an effect algebra $(E, \oplus, \mathbf{0}, \mathbf{1})$ is $\oplus$-orthogonal if for all $n \leq k$ $x_{1} \oplus x_{2} \oplus \cdots \oplus x_{n}=\left(x_{1} \oplus x_{2} \oplus \cdots \oplus x_{n-1}\right) \oplus x_{n}$ exists in $E$ (briefly we will write $\bigoplus_{j=1}^{n} x_{j}$ ). We define also $\oplus \emptyset=\mathbf{0}$

Definition 3 For a lattice $(L, \wedge, \vee)$ and a subset $D \subseteq L$ we say that $D$ is a bifull sublattice of $L$, if and only if for any $X \subseteq D, \bigvee_{L} X$ exists if and only if $\bigvee_{D} X$ exists and $\bigwedge_{L} X$ exists if and only if $\bigwedge_{D} X$ exists, in which case $\bigvee_{L}^{L} X=\bigvee_{D} X$ and $\bigwedge_{L} X=\bigwedge_{D}^{D} X$.

It is known that if $E$ is a distributive effect algebra (i.e., the effect algebra $E$ is a distributive lattice - e.g., if $E$ is an MV-effect algebra) then $C(E)=S(E)$. If moreover $E$ is Archimedean and atomic then the set of atoms of $C(E)=S(E)$ is the set $\left\{n_{a} a ; a \in E\right.$ is an atom of $\left.E\right\}$, where $n_{a}=\operatorname{ord}(a)$ (see [20]). Since $S(E)$ is a bifull sublattice of $E$ if $E$ is an Archimedean atomic LEA (see [13]), we obtain that

$$
\begin{aligned}
\mathbf{1} & =\bigvee_{C(E)}\{p \in C(E) ; p \text { is an atom of } C(E)\} \\
& =\bigvee_{E}\{p \in C(E) ; p \text { is an atom of } C(E)\}
\end{aligned}
$$

for every Archimedean atomic distributive lattice effect algebra $E$. In [8] it was shown that there exists an LEA $E$ for which this property fails to be true. Important properties of Archimedean atomic lattice effect algebras with an atomic center were proven by Riečanová in [21].

Theorem 1 (Riečanová [21]) Let E be an Archimedean atomic lattice effect algebra with an atomic center $C(E)$. Let $A_{E}$ be the set of all atoms of $E$ and $A_{C(E)}$ the set of all atoms of $C(E)$. The following conditions are equivalent:

1. $\bigvee_{E} A_{C(E)}=\mathbf{1}$.

2. For every atom $a \in A_{E}$ there exists an atom $p_{a} \in A_{C(E)}$ such that $a \leq p_{a}$.

3. For every $z \in C(E)$ it holds

$$
\begin{aligned}
z & =\bigvee_{C(E)}\left\{p \in A_{C(E)} ; p \leq z\right\} \\
& =\bigvee_{E}\left\{p \in A_{C(E)} ; p \leq z\right\}
\end{aligned}
$$

4. $C(E)$ is a bifull sub-lattice of $E$.

In this case $E$ is isomorphic to a subdirect product of Archimedean atomic irreducible lattice effect algebras.

Theorem 2 (Paseka, Riečanová [13]) Let E be an atomic Archimedean lattice effect algebra. Then the set $S(E)$ of all sharp elements of $E$ is a bifull sublattice of $E$.

We will deal only with atomic Archimedean lattice effect algebras $E$. We have $C(E) \subset S(E) \subset E$. Because of this inclusion and Theorem 2, considering the bifullness of the center $C(E)$ in $E$ is equivalent to considering the bifullness of $C(E)$ in $S(E)$. And $S(E)$ is an orthomodular lattice. For this reason, in the rest of the paper we will restrict our attention to atomic orthomodular lattices $L$ and their centers $C(L)$. For the sake of completeness, we give the definition of an orthomodular lattice.

Definition 4 Let $L$ be a bounded lattice with a unary operation' (called complementation) satisfying the following conditions

1. for all $a \in L\left(a^{\prime}\right)^{\prime}=a$,

2. for all $a, b \in L$ if $a \leq b$ then $b^{\prime} \leq a^{\prime}$,

3. for all $a, b \in L$ if $a \leq b$ then $a \vee\left(a^{\prime} \wedge b\right)=b$.

Then $L$ is said to be an orthomodular lattice (OML for brevity).

Remark 1 Though in OML's we have just latticetheoretical operations $\vee$ and $\wedge$, we will use also effect algebraic operations $\oplus$ and $\ominus$ with the meaning $a \oplus b=a \vee b$ iff $a \leq b^{\prime}$ and $a \ominus b=c$ iff $b \oplus c=a$. 


\section{Orthomodular lattice $L$ whose center is not a bifull sublattice}

Let us have the following sequences of atoms (sets):

$$
\begin{aligned}
a_{0}= & \left\{(x, y) \in \mathbb{R}^{2} ; 0 \leq x \leq 1, y \in \mathbb{R}\right\}, \\
a_{l}= & \left\{(x, y) \in \mathbb{R}^{2} ; l<x \leq l+1, y \in \mathbb{R}\right\}, \\
& \text { for } l=1,2, \ldots, \\
b_{0}= & \left\{(x, y) \in \mathbb{R}^{2} ;-1 \leq x<0, y \in \mathbb{R}\right\}, \\
b_{l}= & \left\{(x, y) \in \mathbb{R}^{2} ;-l-1 \leq x<-l, y \in \mathbb{R}\right\}, \\
& \quad \text { for } l=1,2, \ldots, \\
c_{j}= & \left\{(x, y) \in \mathbb{R}^{2} ;-j \leq x \leq j, y \leq j \cdot x\right\}, \\
& \quad \text { for } j=1,2, \ldots, \\
d_{j}= & \left\{(x, y) \in \mathbb{R}^{2} ;-j \leq x \leq j, y>j \cdot x\right\}, \\
& \quad \text { for } j=1,2, \ldots, \\
p_{j}= & \{j\}, \quad \text { for } j=1,2, \ldots .
\end{aligned}
$$

For such a choice of atoms, $q_{1} \neq q_{2}$ are compatible if and only if $q_{1} \cap q_{2}=\emptyset$. Fig. 1 shows the compatibility among atoms. For their non-compatibility (denoted by $\leftrightarrow$ ) the following rules hold

$$
\begin{array}{lll}
c_{j} \leftrightarrow a_{i}, \quad c_{j} \leftrightarrow b_{i} & \begin{array}{l}
\text { for all } j=1,2, \ldots \\
\text { and } i=0, \ldots, j-1,
\end{array} \\
d_{j} \leftrightarrow a_{i}, \quad d_{j} \leftrightarrow b_{i} & \text { for all } j=1,2, \ldots \\
& & \text { and } i=0, \ldots, j-1, \\
& \text { for all } i, j=1,2, \ldots \\
c_{j} \leftrightarrow d_{i} & & \text { such that } i \neq j, \\
& & \text { for all } i, j=1,2, \ldots \\
c_{j} \leftrightarrow c_{i}, & d_{j} \leftrightarrow d_{i} & \text { such that } i \neq j .
\end{array}
$$

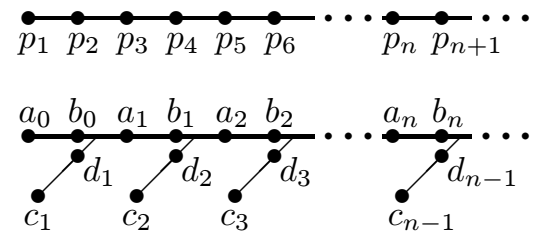

Fig. 1: Greechie diagram of sets of atoms

For non-compatible atoms the following equalities hold

$$
\begin{aligned}
c_{j} \oplus d_{j} & =\bigoplus_{i=0}^{j-1}\left(a_{i} \oplus b_{i}\right) \\
& =c_{k} \vee c_{j}=d_{k} \vee d_{j} \\
& =c_{k} \vee d_{j}=d_{k} \vee c_{j} \\
& =c_{j} \vee a_{l}=c_{j} \vee b_{l} \\
& =d_{j} \vee a_{l}=d_{j} \vee b_{l}
\end{aligned}
$$

for $1 \leq k<j$ and $0 \leq l<j$.
Denote $\hat{B}_{0}, \hat{B}_{j}$ (for $j=1,2, \ldots$ ) complete atomic Boolean algebras with the corresponding sets of atoms $A_{0}, A_{j}(j=1,2, \ldots)$, given by

$$
\begin{aligned}
A_{0}= & \bigcup_{i=0}^{\infty}\left\{a_{i}\right\} \cup \bigcup_{i=0}^{\infty}\left\{b_{i}\right\} \cup \bigcup_{j=1}^{\infty}\left\{p_{j}\right\}, \\
A_{j}= & \bigcup_{i=j}^{\infty}\left\{a_{i}\right\} \cup \bigcup_{i=j}^{\infty}\left\{b_{i}\right\} \cup \bigcup_{j=1}^{\infty}\left\{p_{j}\right\} \\
& \cup\left\{c_{j}, d_{j}\right\} .
\end{aligned}
$$

Disjointness occurring among some atoms of the system (2) is equivalent to the fact that $A_{0}$ and $A_{j}$ $(j=1,2, \ldots)$ are unique maximal sets of pairwise compatible atoms.

Theorem 3 (Kalina [9]) Let $\hat{L}=\bigcup_{i=0}^{\infty} \hat{B}_{i}$. Let $L_{1}$ be the complete OML generated by sets of atoms $\bigcup_{i=0}^{\infty}\left\{a_{i}, b_{i}\right\} \cup \bigcup_{j=1}^{\infty}\left\{c_{j}, d_{j}\right\}$ and $\mathbf{N}$ the complete Boolean algebra generated by the set of atoms $\bigcup_{j=1}^{\infty}\left\{p_{j}\right\}$. Then $(\hat{L}, \vee, \wedge, \mathbf{0}, \mathbf{1})$ is a complete $O M L$ and $\hat{L} \cong L_{1} \times \mathbf{N}$.

An element $u \in \hat{B}_{l}$ is finite if and only if $u=$ $q_{1} \oplus q_{2} \oplus \ldots \oplus q_{n}$ for an $n \in \mathbb{N}$ and $q_{1}, q_{2}, \ldots, q_{n} \in A_{l}$. Set $Q_{l}=\left\{u \in B_{l} ; u\right.$ is finite $\}, l=0,1,2, \ldots$ Then $Q_{l}$ is a generalized Boolean algebra, since $B_{l}=Q_{l} \cup \dot{\cup} Q_{l}^{*}$ is a Boolean algebra, where $Q_{l}^{*}=\left\{u^{*} ; u^{*}=1_{l} \ominus u\right.$ and $\left.u \in Q_{l}\right\}$ (see [22], or [2, pp. 18-19]). This means that $B_{l}$ is a Boolean subalgebra of finite and cofinite elements of $\hat{B}_{l}(l=0,1,2, \ldots)$.

Theorem 4 (Kalina [8]) Denote $L=\bigcup_{l=0}^{\infty} B_{l}$. Then $(L, \vee, \wedge, \mathbf{0}, \mathbf{1})$ is a compactly generated orthomodular lattice with the family $\left(B_{l}\right)_{l=0}^{\infty}$ of atomic blocks of $L$. The center of $L, C(L)$, is not a bifull sublattice of $L$.

\section{Completion of the center of $L$}

We are going to show that it is possible to extend the orthomodular lattice $L$ from Theorem 4 to $\bar{L}$, whose center, $C(\bar{L})$, is a complete Boolean algebra which is not a bifull sublattice of $\bar{L}$.

Denote $\mathcal{F}$ a fixed non-trivial ultrafilter on $\mathbb{N}$ (the index set of atoms $p_{j}$ ). Then $\mathcal{F}$ has the following properties which will be important for our construction:

- Let $F \subset \mathbb{N}$. Then either $F \in \mathcal{F}$ or $\mathbb{N} \backslash F \in \mathcal{F}$.

- Let $F \subset \mathbb{N}$ be a finite set. Then $F \notin \mathcal{F}$.

- If $F_{1} \in \mathcal{F}$ and $F_{2} \in \mathcal{F}$ then $F_{1} \cap F_{2} \in \mathcal{F}$.

- If $F_{1} \in \mathcal{F}$ and $F_{2} \supset F_{1}$ then $F_{2} \in \mathcal{F}$. 
Let $Q_{L_{1}}$ denote the set of all finite elements of $L_{1}$. Further set

$$
P_{\mathcal{F}}=\left\{\bigoplus_{i \in F} p_{i} ; F \notin \mathcal{F}\right\}
$$

and

$$
\begin{aligned}
G & =\left\{f \oplus g ; g \in Q_{L_{1}}, f \in P_{\mathcal{F}}\right\}, \\
G^{\perp} & =\left\{h^{\prime} \in \hat{L} ; h \in G\right\} .
\end{aligned}
$$

Theorem 5 Let $\tilde{L}=G \dot{\cup} G^{\perp}$. Then the system $(\tilde{L}, \vee, \wedge, \mathbf{0}, \mathbf{1})$ is an orthomodular lattice.

The center

$$
C(\tilde{L})=\left\{f \in \tilde{L} ; f \in P_{\mathcal{F}} \text { or } f^{\prime} \in P_{\mathcal{F}}\right\},
$$

and $C(\tilde{L})$ is a complete Boolean algebra which is not bifull in $\tilde{L}$.

Proof. First we show that $\tilde{L}$ is a bounded lattice. Consider elements $h_{1}, h_{2} \in G$. Then there exist elements $g_{1}, g_{2} \in Q_{L_{1}}$ and elements $f_{1}, f_{2} \in P_{\mathcal{F}}$ such that

$$
h_{1}=f_{1} \oplus g_{1}, \quad h_{2}=f_{2} \oplus g_{2} .
$$

By the properties of the non-trivial ultrafilter $\mathcal{F}$ we get that $f_{1} \vee f_{2} \in P_{\mathcal{F}}$ and $f_{1} \wedge f_{2} \in P_{\mathcal{F}}$. Since $g_{1}, g_{2}$ are finite elements of $L_{1}$, we get that $g_{1} \vee g_{2} \in Q_{L_{1}}$ and also $g_{1} \wedge g_{2} \in Q_{L_{1}}$. Since $L_{1}$ is generated by the sets of atoms $\bigcup_{i=0}^{\infty}\left\{a_{i}, b_{i}\right\}$ and $\bigcup_{j=1}^{\infty}\left\{c_{j}, d_{j}\right\}$, each $g \in Q_{L_{1}}$ is $\oplus$-orthogonal to each $f \in P_{\mathcal{F}}$. This implies that $G$ is closed under $\vee$ and $\wedge$. Because $G^{\perp}$ consists of complements of elements of $G$, we have that also $G^{\perp}$ is closed under $\vee$ and $\wedge$. Now assume that $h_{1} \in G$ and $h_{2} \in G^{\perp}$. Then $h_{2}^{\prime} \in G$ and we can write

$$
h_{1}=f_{1} \oplus g_{1}, \quad h_{2}^{\prime}=f_{2} \oplus g_{2}
$$

with the same meaning of $f_{1}, f_{2}, g_{1}, g_{2}$ as in formula (6). This means that $h_{2}=f_{2}^{\prime} \ominus g_{2}$. Then, because of the monotonicity of the ultrafilter $\mathcal{F}$, we have $\left(f_{1} \vee f_{2}^{\prime}\right)^{\prime} \in P_{\mathcal{F}}$ and hence $f_{1} \vee f_{2}^{\prime} \in G^{\perp}$. Moreover, $g_{2} \in Q_{L_{1}}$ is orthogonal to $f_{1}$ which implies

$$
\left(f_{1} \vee f_{2}^{\prime}\right) \ominus g_{2}=f_{1} \vee\left(f_{2}^{\prime} \ominus g_{2}\right) \in G^{\perp} .
$$

Since $G$ is a monotone system (meaning that with an arbitrary element $\delta_{1} \in G$ it contains also all elements $\delta_{2} \in \hat{L}$ such that $\delta_{2} \leq \delta_{1}$ ), we get from the duality between $G$ and $G^{\perp}$ that

$$
\left(f_{1} \vee g_{1}\right) \vee\left(f_{2}^{\prime} \ominus g_{2}\right)=h_{1} \vee h_{2} \in G^{\perp}
$$

Dually we get that $h_{1} \wedge h_{2} \in G$. This implies that $\tilde{L}=G \dot{\cup} G^{\perp}$ is a lattice. Obviously it is a bounded and orthocomplemented lattice. Showing that it is an OML is a matter of routine. We will omit the detailed proof.
Let us consider an element $f \in \tilde{L}$ such that $f \in P_{\mathcal{F}}$ or $f^{\prime} \in P_{\mathcal{F}}$. Then $f$ is a central element. If $f$ is such that neither $f \in P_{\mathcal{F}}$ nor $f^{\prime} \in P_{\mathcal{F}}$, then there exist atoms $\alpha_{1}, \alpha_{2} \in \bigcup_{i=0}^{\infty}\left\{a_{i}, b_{i}\right\} \cup \bigcup_{j=1}^{\infty}\left\{c_{j}, d_{j}\right\}$ fulfilling $\alpha_{1} \leftrightarrow \alpha_{2}$ and $\alpha_{1} \leq f, \alpha_{2} \not \leq f$. Then $f$ is not a central element. This proves that

$$
C(\tilde{L})=\left\{f \in \tilde{L} ; f \in P_{\mathcal{F}} \text { or } f^{\prime} \in P_{\mathcal{F}}\right\}
$$

Due to the fact that $\mathcal{F}$ is a non-trivial ultrafilter, $C(\tilde{L})$ is a complete Boolean algebra.

The only central element that is greater than all atoms $p_{j}$ for $j=1,2, \ldots$, is $\mathbf{1}$, hence we have that

$$
\bigvee_{C(\tilde{L})}\left\{p_{j} ; j=1,2, \ldots\right\}=\mathbf{1}
$$

On the other hand, let us take an arbitrary atom $\alpha \in$ $\bigcup_{i=0}^{\infty}\left\{a_{i}, b_{i}\right\} \cup \bigcup_{j=1}^{\infty}\left\{c_{j}, d_{j}\right\}$ and assume that $\bigvee_{\tilde{L}}\left\{p_{j} ; j=\right.$ $1,2, \ldots\}$ does exist. Since $\alpha$ is orthogonal to all atoms from the set $\bigcup_{j=1}^{\infty}\left\{p_{j}\right\}$, we have that $\alpha$ is orthogonal to $\bigvee_{\tilde{L}}\left\{p_{j} ; j=1,2, \ldots\right\}$ and hence

$$
\bigvee_{\tilde{L}}\left\{p_{j} ; j=1,2, \ldots\right\} \neq \mathbf{1}
$$

It can be shown (see [8]) that $\bigvee_{\tilde{L}}\left\{p_{j} ; j=1,2, \ldots\right\}$ does not exist. This implies that $C(\tilde{L})$ is not a bifull sublattice of $\tilde{L}$.

\section{$4 \sigma$-complete orthomodular lattice $\tilde{L}_{\sigma}$ whose center is not a bifull sublattice}

Let $\mathcal{I}$ denote the set of all ordinal numbers less than $\Omega$ (the first uncountable ordinal number). Further, denote $\mathcal{E}$ the set of all limit ordinal numbers up to $\Omega$ and $\mathcal{J}=\mathcal{I} \backslash \mathcal{E}$.

Assume sets of elements $\left\{p_{i} ; i \in \mathcal{I}\right\},\left\{a_{i} ; i \in \mathcal{I}\right\}$, $\left\{b_{i} ; i \in \mathcal{I}\right\},\left\{c_{i} ; i \in \mathcal{I}\right\},\left\{d_{i} ; i \in \mathcal{I}\right\}$, where the corresponding elements for $i \in \mathcal{J}$ will act as atoms. We will have a partial relation $\Varangle$ modelling noncompatibility. This partial relation will have the following form among atoms

$c_{j} \nLeftarrow a_{i}, \quad c_{j} \nLeftarrow b_{i} \quad$ for all $j \in \mathcal{J}$ and $i \leq j$, $d_{j} \leftrightarrow a_{i}, \quad d_{j} \leftrightarrow b_{i} \quad$ for all $j \in \mathcal{J}$ and $i \leq j$, 


$$
\begin{array}{cc}
c_{j} \leftrightarrow d_{i} & \begin{array}{r}
\text { for all } \quad i, j \in \mathcal{J} \\
\text { such that } i \neq j,
\end{array} \\
c_{j} \leftrightarrow c_{i}, \quad d_{j} \leftrightarrow d_{i} \quad \begin{array}{l}
\text { for all } \quad i, j \in \mathcal{J} \\
\text { such that } i \neq j .
\end{array}
\end{array}
$$

Sets of elements $\left\{p_{i} ; i \in \mathcal{I}\right\},\left\{a_{i} ; i \in \mathcal{I}\right\},\left\{b_{i} ; i \in \mathcal{I}\right\}$, $\left\{c_{i} ; i \in \mathcal{I}\right\},\left\{d_{i} ; i \in \mathcal{I}\right\}$ will present atoms for $i \in \mathcal{J}$ and for $\kappa \in \mathcal{E}$ we will have

$$
\begin{aligned}
p_{\kappa} & =\bigvee_{i<\kappa} p_{i}, \\
a_{\kappa} & =\bigvee_{i<\kappa} a_{i}, \\
b_{\kappa} & =\bigvee_{i<\kappa} b_{i}, \\
c_{\kappa} & =\bigvee_{i<\kappa} c_{i}=\bigvee_{i<\kappa} d_{i}=d_{\kappa}=a_{\kappa} \oplus b_{\kappa} .
\end{aligned}
$$

As a possible model for the just presented sets of elements fulfilling the non-compatibility relation we may have the following:

Let us choose a good order of positive real numbers of type $\Omega$, i.e., positive real numbers will be enumerated by ordinal numbers from $\mathcal{J}$. For $i \in \mathcal{J}$ and $r>0$, $r \in \mathbb{R}$, we denote $r_{i}$ the $i$-th number in the chosen good order. Then we identify the set $\left\{p_{i} ; i \in \mathcal{J}\right\}$ with the set of all positive real numbers, i.e., $p_{i}=r_{i}$. Further we put for $i, j \in \mathcal{J}$

$$
\begin{aligned}
a_{i} & =\left\{\left(r_{i}, y\right) \in \mathbb{R}^{2} ; y \in \mathbb{R}\right\}, \\
b_{i} & =\left\{\left(-r_{i}, y\right) \in \mathbb{R}^{2} ; y \in \mathbb{R}\right\}, \\
c_{i} & =\left\{\left(r_{j}, y\right) \in \mathbb{R}^{2} ; j \leq i, y \leq r_{i} \cdot r_{j}\right\} \\
& \cup\left\{\left(-r_{j}, y\right) \in \mathbb{R}^{2} ; j \leq i, y \leq-r_{i} \cdot r_{j}\right\}, \\
d_{i} & =\left\{\left(r_{j}, y\right) \in \mathbb{R}^{2} ; j \leq i, y>r_{i} \cdot r_{j}\right\} \\
& \cup\left\{\left(-r_{j}, y\right) \in \mathbb{R}^{2} ; j \leq i, y>-r_{i} \cdot r_{j}\right\} .
\end{aligned}
$$

For $\kappa \in \mathcal{E}$ we define the corresponding elements $p_{\kappa}, a_{\kappa}, b_{\kappa}, c_{\kappa}, d_{\kappa}$ by equalities $7,8,9,10$, respectively. Compatibility among different atoms is given by disjointness of the corresponding sets. This implies that the uniquely given maximal sets of pairwise compatible atoms are

$$
\begin{aligned}
\tilde{A}_{0}= & \bigcup_{i \in \mathcal{J}}\left\{a_{i}, b_{i}, p_{i}\right\} \\
\tilde{A}_{j}= & \bigcup_{i \in \mathcal{J}}\left\{a_{i}, b_{i}\right\} \cup \bigcup_{i \in \mathcal{J}}\left\{p_{i}\right\} \cup\left\{c_{j}, d_{j}\right\} \\
& i>j
\end{aligned}
$$

for $j \in \mathcal{J}$. Sets of atoms $\tilde{A}_{0}$ and $\tilde{A}_{j}$ for $j \in \mathcal{J}$, generate complete Boolean algebras $\tilde{B}_{0}$ and $\tilde{B}_{j}$ for $j \in \mathcal{J}$, respectively. For $\kappa \in \mathcal{E}$ we get complete atomic
Boolean algebras $\tilde{B}_{\kappa}$ generated by sets of atoms

$$
\begin{aligned}
\tilde{A}_{\kappa}=\bigcup_{i \in \mathcal{J}}\left\{p_{i}\right\} \cup\left\{a_{\kappa}, b_{\kappa}\right\} \cup & \bigcup \\
i & \in \mathcal{J} \\
i & >\kappa
\end{aligned}
$$

This means that for $\kappa \in \mathcal{E} \tilde{B}_{\kappa} \subset \tilde{B}_{0}$. The union of all complete atomic Boolean algebras, $\tilde{L}=\tilde{B}_{0} \cup \bigcup_{i \in \mathcal{I}} \tilde{B}_{i}$, is a complete OML. An element $f \in \tilde{L}$ will be called countable if there exists an at most countable set of atoms (an at most countable set of indices $K$ ) $\left\{q_{k}\right\}_{k \in K} \subset \tilde{A}_{0}$ or $\left\{q_{k}\right\}_{k \in K} \subset \tilde{A}_{i}$ for $i \in \mathcal{J}$, such that

$$
f=\bigoplus_{k \in K} q_{k} .
$$

By definition of elements $p_{i}, a_{i}, b_{i}, c_{i}, d_{i}$ for $i \in \mathcal{I}$ we get that each of these elements is countable.

Let $\mathcal{K}$ denote the set of all countable elements of $\tilde{L}$ and $\mathcal{K}^{\perp}=\left\{f \in \tilde{L} ; f^{\prime} \in \mathcal{K}\right\}$. Further, let $\mathcal{P}$ denote the set of all countable elements generated by $\left\{p_{i}, i \in \mathcal{J}\right\}$, and $\mathcal{P}^{\perp}=\left\{f \in \tilde{L} ; f^{\prime} \in \mathcal{P}\right\}$.

Theorem 6 Let $\tilde{L}_{\sigma}=\mathcal{K} \cup \mathcal{K}^{\perp}$. Then $\left(\tilde{L}_{\sigma}, \vee, \wedge, \mathbf{0}, \mathbf{1}\right)$ is a $\sigma$-complete OML. The center $C\left(\tilde{L}_{\sigma}\right)=\mathcal{P} \dot{\cup} \mathcal{P}^{\perp}$ and it is not a bifull sublattice of $\tilde{L}_{\sigma}$.

Proof. Each of the atoms $p_{i}, a_{i}, b_{i}, c_{i}, d_{i}$ for $i \in \mathcal{J}$ (and hence also each of the elements $p_{i}, a_{i}, b_{i}, c_{i}, d_{i}$ for $i \in \mathcal{I}$ ) is countable. This implies that $\tilde{L}_{\sigma}$ is an OML. Since it is by definition closed under countable meets and joins, it is $\sigma$-complete.

Elements $p_{i}$ for $i \in \mathcal{I}$ are central because each of the elements $p_{i}$ is compatible with all atoms of $\tilde{L}_{\sigma}$. This implies that $\mathcal{P} \dot{\cup} \mathcal{P}^{\perp} \subset C\left(\tilde{L}_{\sigma}\right)$. On the other hand, let $f$ be a countable element, $f \notin \mathcal{P}$. Then there exists $c_{i}$ such that $c_{i} \not \leq f$ for $i \in \mathcal{J}$ and an atom out of $e \in\left\{a_{j}, b_{j}, c_{j}, d_{j}\right\}$ for $j<i, e \leq f$. Then $c_{i} \leftrightarrow e$ and hence $c_{j} \leftrightarrow f$. Similarly, if $f \in \mathcal{K}^{\perp}$, there exists $c_{i} \leq f$ and an atom out of $e \in\left\{a_{j}, b_{j}, c_{j}, d_{j}\right\}$ for $j<i$ such that $e \not \leq f$. In this case $e \not t c_{i}$ and hence also $e \leftrightarrow f$. We conclude that $C\left(\tilde{L}_{\sigma}\right)=\mathcal{P} \dot{\cup} \mathcal{P}^{\perp}$.

We show that $C\left(\tilde{L}_{\sigma}\right)$ is not a bifull sublattice of $\tilde{L}_{\sigma}$. Obviously

$$
\bigvee_{C\left(\tilde{L}_{\sigma}\right)}\left\{p_{i}, i \in \mathcal{I}\right\}=\mathbf{1} .
$$

Assume that $\bigvee_{\tilde{L}_{\sigma}}\left\{p_{i}, i \in \mathcal{I}\right\}$ does exist. Then all elements $e \in \bigcup_{i \in \mathcal{I}}\left\{a_{i}, b_{i}, c_{i}, d_{i}\right\}$ are orthogonal with all elements from the set $\bigcup_{\mathcal{I}}\left\{p_{j}\right\}$ and consequently also with $\bigvee_{\tilde{L}_{\sigma}}\left\{p_{i}, i \in \mathcal{I}\right\}$. This implies $\bigvee_{\tilde{L}_{\sigma}}\left\{p_{i}, i \in \mathcal{I}\right\} \neq \mathbf{1}$. This means that $C\left(\tilde{L}_{\sigma}\right)$ is not a bifull sublattice of $\tilde{L}_{\sigma}$. 


\section{Acknowledgement}

Support from the Science and Technology Assistance Agency under contract No. APVV-0073-10, and from the VEGA grant agency, grant number $1 / 0297 / 11$, is gratefully acknowledged.

\section{References}

[1] Chang, C. C.: Algebraic analysis of many-valued logics. Trans. Amer. Math. Soc. 88 (1958), 467-490.

[2] Dvurečenskij, A., Pulmannová, S.: New Trends in Quantum Structures. Dordrecht, Boston, London, and Isterscience, Bratislava : Kluwer Acad. Publisher, 2000.

[3] Foulis, D. J., Bennett, M. K.: Effect algebras and unsharp quantum logics. Found. Phys. 24 (1994), 1325-1346.

[4] Greechie, R. J., Foulis, D. J., Pulmannová, S.: The center of an effect algebra. Order 12 (1995), 91-106.

[5] Gudder, S. P.: Sharply dominating effect algebras. Tatra Mountains Math. Publ. 15 (1998), 23-30.

[6] Gudder, S. P.: S-dominating effect algebras. Internat. J. Theor. Phys. 37 (1998), 915-923.

[7] Jenča, G., Riečanová, Z.: On sharp elements in lattice ordered effect algebras. BUSEFAL 80 (1999), 24-29.

[8] Kalina, M.: On central atoms of Archimedean atomic lattice effect algebras. Kybernetika 46 (2010), 4, 609-620.

[9] Kalina, M.: Mac Neille completion of centers and centers of Mac Neille completions of lattice effect algebras. Kybernetika 46 (2010), 6, 635-647.

[10] Kôpka, F.: Compatibility in D-posets. Internat. J. Theor. Phys. 34 (1995), 1525-1531.

[11] Mosná, K.: About atoms in generalized efect algebras and their effect algebraic extensions. J. Electr. Engrg. 57 (2006), 7/s, 110-113.
[12] Mosná, K., Paseka, J., Riečanová, Z.: Order convergence and order and interval topologies on posets and lattice effect algebras. In Proc. internat. seminar UNCERTAINTY 2008, Publishing House of STU 2008, 45-62.

[13] Paseka, J., Riečanová, Z.: The inheritance of BDE-property in sharply dominating lattice effect algebras and (o)-continuous states. Soft Computing, 15 (2011), 543-555.

[14] Riečanová, Z.: Compatibility and central elements in effect algebras. Tatra Mountains Math. Publ. 16 (1999), 151-158.

[15] Riečanová, Z.: Subalgebras, intervals and central elements of generalized effect algebras. Internat. J. Theor. Phys., 38 (1999), 3 209-3220.

[16] Riečanová, Z.: Generalization of blocks for Dlattices and lattice ordered effect algebras. Internat. J. Theor. Phys. 39 (2000), 231-237.

[17] Riečanová, Z.: Orthogonal sets in effect algebras. Demontratio Mathematica 34 (2001), $525-532$.

[18] Riečanová, Z.: Smearing of states defined on sharp elements onto effect algebras. Internat. $J$. Theor. Phys. 41 (2002), 1511-1524.

[19] Riečanová, Z.: Subdirect decompositions of lattice effect algebras. Internat. J. Theor. Phys. 42 (2003), 1425-1433.

[20] Riečanová, Z.: Distributive atomic effect akgebras. Demontratio Mathematica 36 (2003), $247-259$.

[21] Riečanová, Z.: Lattice effect algebras densely embeddable into complete ones. Kybernetika, $\mathbf{4 7}$ (2011), 1, 100-109.

[22] Riečanová, Z., Marinová, I.: Generalized homogenous, prelattice and MV-effect algebras. Kybernetika 41 (2005), 129-142.

Martin Kalina

E-mail: kalina@math.sk

Dept. of Mathematics

Faculty of Civil Engineering

Slovak Univ. of Technology

Radlinského 11, Sk-813 68 Bratislava, Slovakia 